\title{
Traumatic Hepatic artery Transection Reconstructed with Splenic Artery Transposition Graft
}

\author{
Pil Young Jung, Seong yup Kim, Keum Seok Bae \\ Department of Surgery, Yonsei university Wonju college of medicine, Wonju Severance Christian Hospital, Trauma center
}

Traumatic injury of the hepatic artery is not common. However, it is a critical and lethal injury because of a higher risk of complication. Several methods for reconstruction of the hepatic artery have been described, including interposition of venous and prosthetic grafts, and transposition of native arteries to the distal stump of the hepatic artery. We describe a case of transposition to reconstruct the hepatic artery using the splenic artery.

Key Words: Hepatic Artery; Injuries; Splenic Artery

(Trauma Image Proced 2017(1):25-26)

\section{CASE}

A 61-year-old male patient with a history of hypertension was admitted via the emergency room with

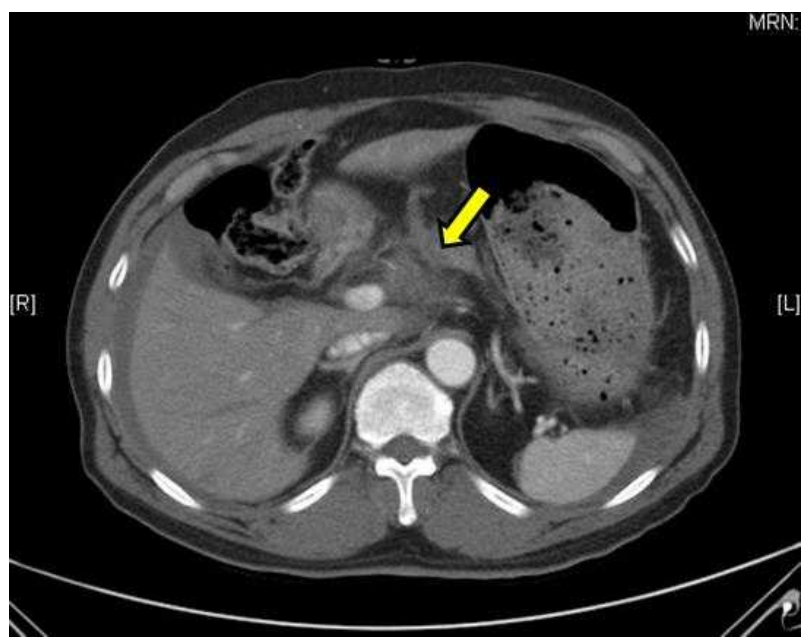

Fig. 1. Abdominal CT shows ongoing bleeding of the common hepatic artery (yellow arrow) with hemoperitoneum. blunt trauma. At admission, he was in shock status and had a positive focused abdominal sonography for trauma (FAST) sign. Computed tomography (CT) of the abdomen-pelvis confirmed a hemoperitoneum and minor pancreatic injury (Fig. 1.). Therefore, emergency surgery was performed for the hemoperitoneum and detachment of the gastroduodenal artery from the common hepatic artery was found. Accordingly, the hepatic artery was reconstructed with a splenic artery transposition graft (Fig. 2.). After 10 days, a follow-up CT scan revealed normal perfusion status of the liver (Fig. 3.). The patient recovered and was discharged without complications.

\section{DISCUSSION}

Hepatic artery transection presents a technical challenge in vascular reconstruction. Various strategies to manage a hepatic artery injury have been described,

Received: March 12, 2017 Revised: April 3, 2017 Accepted: April 3, 2017

Correspondence to: Keum Seok Bae, Department of Surgery, Yonsei university Wonju college of medicine, Wonju Severance Christian Hospital, 65, 220-701, 20 IIsan-ro, Wonju-si, Gangwon-do, Korea

Tel: 82-33-741-0881, Fax: 82-33-741-1205, E-mail: bksgs@yonsei.ac.kr

Copyright (c) 2017 Korean Association for Research, Procedures and Education on Trauma. All rights reserved.

(c)This is an open-access article distributed under the terms of the Creative Commons Attribution Non-Commercial License (http://creativecommons.org/ licenses/by-nc/4.0) which permits unrestricted noncommercial use, distribution, and reproduction in any medium, provided the original work is properly cited 


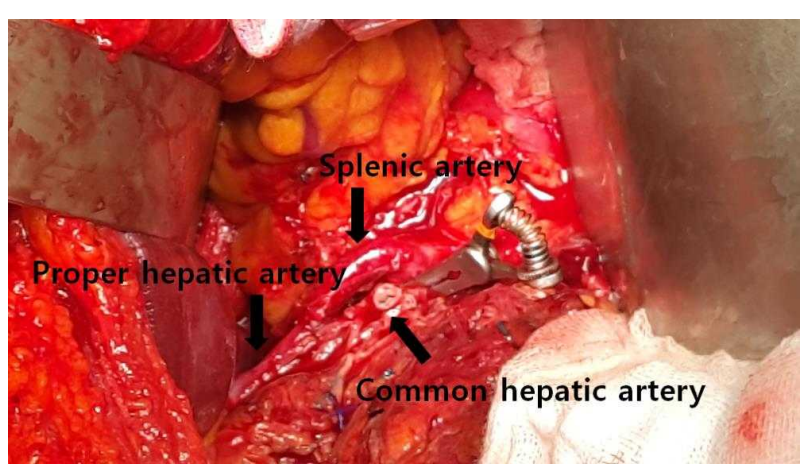

Fig. 2. Reconstruction of the hepatic artery with a splenic artery transposition graft

ranging from ligation to complex vascular reconstruction (1). Injuries to the hepatic arteries are uncommon in experienced hands (2). In conclusion, splenic artery transposition is a simple and safe surgical option in patients with traumatic hepatic artery injury.

\section{Conflict of Interest Statement}

No potential conflict of interest relevant to this article was reported.

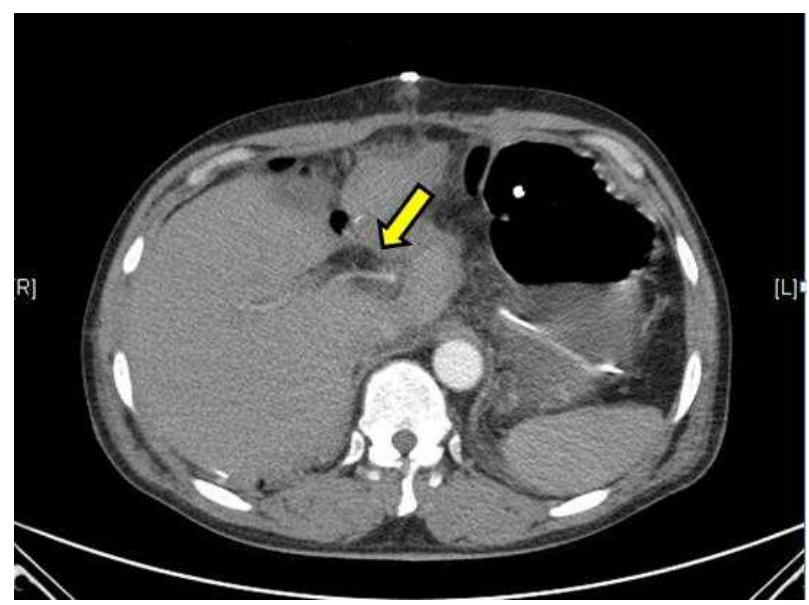

Fig. 3. Follow-up CT reveals normal perfusion status of the liver (yellow arrow).

\section{REFFERENCES}

1. Faulds J, Johner A, Klass D, Buczkowski A, Scudamore $\mathrm{CH}$. Hepatic artery transection reconstructed with splenic artery transposition graft. Perspectives in vascular surgery and endovascular therapy. 2012;24(2):87-9.

2. Kulkarni GV, Malinowski M, Hershberger R, Aranha GV. Proper hepatic artery reconstruction with gastroduodenal artery transposition during pancreaticoduodenectomy. Perspectives in vascular surgery and endovascular therapy. 2013;25(3-4):69-72. 